\title{
LES ARMÉES FRANÇAISES LORS \\ DE LA GUERRE DE CENT ANS
}

\author{
PAR \\ J.F. FINÓ
}

L'ÉTUDE de l'art militaire est fort interéssante quelque soit l'époque envisagée, mais cette étude devient passionnante lorsqu'il s'agit de l'Antiquité ou du Moyen Âge. Elle fournit alors de précieux enseignements sur les institutions et sur leurs applications, sur l'état des techniques et sur les réalisations qui on été faites, sur le chiffre de la population, etc. Faire la guerre êtant naturel chez les hommes, on ne peut juger des moyens dont ils se sont servis pour attaquer leurs adversaires sans faire un examen complet de tout ce qui a constitué la vie de ces hommes. Le champ ainsi couvert est immense mais, dans les pages qui suivent, on se bornera à considérer un épisode de l'histoire de France aux $\mathrm{XIV}^{\mathrm{c}}$ et $\mathrm{XV}^{\mathrm{e}}$ siècles, propos qui sera encore limité du fait que l'on n'insistera quelque peu que sur ce qui se rapporte aux armes.

\section{LA SCÈnE POLITIQUe ET TECHNIQUE}

On a dit avec raison «Une armée, un système militaire, sont toujours organisés, conçus, dirigés contre un ou plusieurs adversaires, au moins virtuels, qu'il s'agisse de les attaquer ou de s'en défendre» ${ }^{1}$. Dans le cas présent, l'ennemi c'est l'Angleterre dont les rois sont, notamment et à la fois, le vassal (pour la Guyenne, etc.), l'adversaire économique (pour les laines fournies aux tisserants flamands) et le rival dynastique (par les droits des femmes) des Valois qui occupent le trône de France. Donc, une étude, même sommaire, des armées françaises de cette époque ne peut guère être entreprise sans avoir présent à l'esprit le contexte politique de la guerre de Cent ans ${ }^{2}$ et sans faire de fréquentes comparaisons avec les réalisations militaires anglaises.

Pour ce qui est des techniques, les $\mathrm{XIV}^{\mathrm{c}}$ et $\mathrm{XV}^{\mathrm{e}}$ siècles apparaissent

' Ph. Contamine: Guerre, État et société à la fin du Moyen Âge; études les armées des rois de France 1337-1494, Paris-La Haye, Mouton, 1972, xxxviii, 757 p. (Civilisations et Sociétés, 24). Cf. c.-r., in: Gladius, t. X, 1972, pp. 92-94.

${ }^{2}$ Ph. Contamine: La guerre de Cent ans, $2^{c}$ éd., Paris, Presses Universitaires de France, 1972, 128 p. (Coll. Que sais-je? n. 1309). Ed. Perroy, La guerre de Cent ans, Paris, Gallimard, 1943, 343 p. (La suite des temps, 13). 
marqués de nombreux progrès ${ }^{3}$. Indiquons-en quelques uns ${ }^{4}$. Large emploi de l'eau comme source d'énergie et les roues à aubes entrainent non seulement des moulins à grains, mais des moulins à tan, des métiers à tisser, de lourds marteaux à battre le fer, de grands soufflets pour insufler l'air dans les forges, etc. Le nombre de machines est devenu plus considérable et l'utilisation du courant des rivières plus savante. Il se produit une véritable concentration industrielle: le nombre total des exploitations diminue mais celles qui subsistent deviennent plus grandes afin de satisfaire aux besoins d'une demande accrue et on assiste ainsi à un ample développement des ressources énergétiques ${ }^{5}$. Pour les transports, l'avant-train mabile apparait en Europe au XIV ${ }^{\mathrm{e}}$ siècle; c'est un organe essentiel des voitures ou des chars auxquels il permet d'évoluer sans être obligés de déraper sur leurs roues au risque de les rompre. Parmis les mécanismes nouveaux, signalons le système biellemanivelle et le cric. Le premier permet de transformer un mouvement rectiligne alternatif en un autre circulaire continu ou vice-versa (Fig. 1) et n'a dû être connu qu'au début du XVe siècle. En effet, si bien on le voit dans le Bellifortis de l'ingénieur allemand Konrad Kyeser, rédigé vers 1390-1405, il ne figure que dans les manuscrits les plus récents de cette oeuvre et il s'agit, peut-être, d'une interpolation. Par contre, dans le carnet de notes d'un autre ingénieur, le manuscrit dit «de la guerre hussiste», qui date des environ de 1430 , il est représenté d'une façon certaine ${ }^{6}$. Quant

"Bertrand Gille: Les ingénieurs de la Renaissance, Paris, Hermann, 1964, 239 p. Cf. c.-r., in: Gladius, t. IV, 1965, pp. 113-115. Un catalogue des manuscrits d'ingénieur de cette époque est dressé, pp. 233-239. Traduction anglaise par L. Humphries, London, 1966.

+ Histoire générale des techniques, dir. M. Daumas, Paris, Presses Univers. de France, 1962 et suiv., 5 vols. Surtout les vols. I et II; $A$ bistory of technology, dir. Ch. Singer, etc., Oxford, Clarendon Press, 1954-1958, 5 vols. Surtout le vol. II.

5 A. M. BAUTIER: Les plus anciennes mentions de moulins bydrauliques industriels et de moulins à vent, in: «Bulletin philologique et historique, jusqu'à 1610 , 1960 , pp. $588-593$.

' Le Bcllifortis, dont des manuscrits se trouvent répartis dans diverses bibliothèques d'Europe, a été étudié à plusieurs reprises, notamment par MARCELLIN BERTHFLOT, Le livre d'un ingénieur militaire à la fin du XIV siècle..., in: «Journal des Savants», 1900 , pp. $1-15$ et $85-94$. Écrit en latin, il a été édité facsimilairement avec version allemande, par Gotz Quarg (London, Phaidon Press, 1968) en 2 vols., d'après le manuscrit de la Bibliot. Universit. de Göttingen, codex philosoph. 63. Nombre de miniatures de ce codex on été reproduites par Marcellin BerTHelot: Histoire des macbines de guerre et des arts mécaniques au Moyen Âge; le livre d'un ingénieur militaire à la fin du XIV siècle, in: «Annales de chimie et de physique», 7 série, t. XIX, 1900, pp. 289-415. Le manuscrit dit «de la guerre hussiste», écrit en vieil allemand, forme la première partie du ms. 197 de la Bayerische Staatsbibliot. de Munich. Un choix de ses miniatures a été donné par MARCellin Berthelot: Pour l'bistoire des arts mécaniques et de l'artillerie vers la fin 
au cric, crémaillère entrainée par la rotation d'un engrenage, il apparait aussi au $X^{*}$ siècle et, au début, il n'est guère utilisé que pour tendre certaines arbalètes.
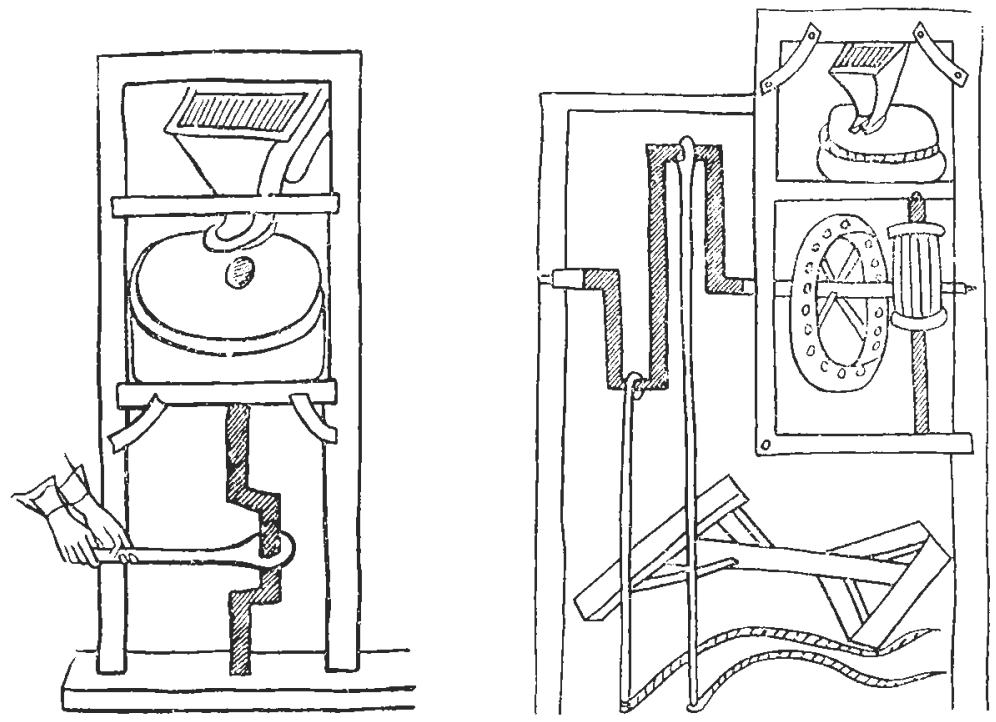

FIG. 1.-Systeme bielle-manivelle. D'après le manuscrit dit «de la guerre hussiste» (n." 197 de la Bayerische Staatsbibliot. de Munich, fol. 18). A gauche, un petit moulin à farine, mû à la main comme nos moulins à café. $A$ droite, un modèle plus grand entrainé par l'effort exercé sur des cables et des brancards. ( $A$ hist. of technol., vol. II, p. 653.)

\section{L'ARMEMENT AU XIV ${ }^{\mathrm{c}}$ SIÈCLE $^{7}$}

Cavalerie.-Au cours des siècles précédents, on êtait passé de la broigne carolingienne, écailles en os, cuir ou métal, superposées comme

du Moyen Âge, in: «Annales de chimie et de physique», $6^{\circ}$ série, t. XXIV, 1891, pp. 433.521 .

- Camilite Enlart: Manuel d'archéologie française depuis les temps mérovingiens jusqu'à la Renaissance, vol. III (Paris, Picard, 1916), pp. 447-526; J.-F. Finó: Forteresses de la France médiévale; construction, attaque, défense, $2^{\mathrm{e}}$ éd., Paris,

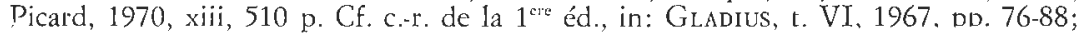
Paul Martin: Armes el armures de Charlemagne à Louis XIV, Paris, Bibliothèque des Arts, 1967, 296 p. Cf. c.-r., in: Gladius, t. X, 1972, pp. 104-106; Vesey Nor- 
les tuiles d'un toit, aux mailles pleines, piècettes de fer fixées sur un fort vêtement, puis aux mailles creuses, petits anneaux entrelacés. Ces dernières forment un tissu, impénétrable aux projectiles, qui couvre le corps ${ }^{8}$ et, pour amortir les chocs, on porte le gambison ou gamboisson, tunique matelassée, similaire à l'escaupil utilisé par les Conquistadors espagnols au $\mathrm{XVI}^{\mathrm{e}}$ siècle pour se défendre contre les flèches des indiens ${ }^{9}$.

Une sorte d'évolution inverse a lieu alors. Des le XIII ${ }^{c}$ siècle avancé, les parties sensibles du corps (épaules, bras, jambes) sont protégées des chocs au moyen de plates, plaquettes rigides de cuir, puis de métal, posées par-dessus le tissu de mailles. Au cours du XIVe siècle, les plates éliminent les mailles sous-jacentes et suffisent à assurer une bonne protection contre les armes adversaires, que celles-ci soient contondantes ou de taille ou d'estoc. Les réalisations sont fort diverses et on n'en indiquera que quelques unes. Les jacques d'écailles, tels ceux qu'exhibe le Musée de la Porte de Hal à Bruxelles, sont formés de petites pièces de fer d'environ 0,03 à $0,04 \mathrm{~m}$. de côté chacune, imbriquées entre-elles et constituant un vêtement dont le poids est, à peu près, de $5 \mathrm{~kg}$. La brigandine ou brigantine, qui fait son apparition à la fin du XIII ${ }^{e}$ siècle, est une sorte de gilet où des plaques et des cercles d'acier, placés entre deux pièces de toile ou de cuir, sont fixés par des clous dont les larges caboches, parfois taillées à pans coupés et dorées ou argentées, font saillie à l'extérieur et forment des dessins plus ou moins géométriques. La brigandine, fort souple et offrant une bonne défense contre les chocs, sera adoptée par beaucoup de grands seigneurs qui en font leur vêtement de guerre favori, comme le prouvent les collections de divers musées d'Europe. Mais, elle est portée aussi par des piétons qui en prendront le nom de brigandiniers ou brigands. Peu avant la guerre de 1914, la Manufacture Française d'armes et cycles de Saint-Etienne (Manufrance) crée le "protège-corps invulnérable» qui s'inspire d'un principe analogue, principe que l'on retrouve aussi dans les gilets pare-balles dont disposent les armées et les polices modernes pour se protéger contre les éclats d'obus (responsablc du $80 \%$ des blessures reçues au sol ou en avion) et contre les projectiles

Man: Arms and armour, London, Weindenfeld and Nicholson, 1964, 127 p. (Pleasures and treasures). Cf. c.-r., in: Gladius, t. IV, 1965, pp. 115-116. Adaptation française par R. J. Charles et traduction par J. Cl. Rolzot, Paris, Hachette, 1966; Eugène Viollet-Le-Duc: Dictionnaire raisonné du mobilier français de l'époque carlovingienne à la Renaissance, Paris, Bance, 1858-1875, 6 vols., Surtout les vols. $\mathrm{V}$ et VI.

${ }^{*}$ Françols Buttin: Du costume militaire au Moyen Age et pendant la Renaissance, Barcelona, Real Academia de Buenas Letras, 1971, 419 p. (Memorias de la R. A. de B. L., XII.)

9 Al.Berto M. Salas: Las ammas de la Conquista, Buenos Aires, Emecé, 1950. pp. 250-257 et 266-26\%. 
des armes légères, tels les pistolets-mitrailleurs ${ }^{10}$. Enfin, lors des fouilles effectuées en Suède sur le champ de la bataille de Wisby, livrée en 1361, on a retrouvé de nombreuses armures, aujourd'hui déposées, pour la plupart, au Musée National de Stockholm. Ces armures apparaissent formée de pièces métallique séparées qui, parfois, peuvent n'être que de simples écailles (les armures s'apparentant ainsi aux jacques belges) tandis que,

Fic. 2.-Chevalicr du début du XIV" siècle. Sceau de Louis comte de Nevers, 1315 (Archives nationales, cote D 876). Le heaume couvre entièrement la tête. Des plates (ailettes et grèves) renforcent le vêtement de mailles entrelacées; l'écu, de dimensions réduites, est armoirié. Une chaînette retient l'épéc. Le cheval eut couvert par une housc. (Phot. Archives nationales.)

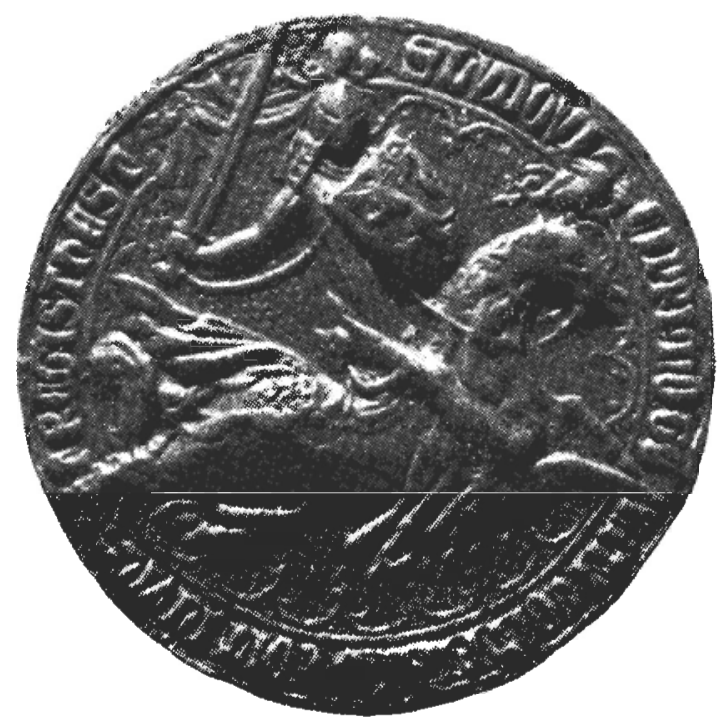

dans d'autres cas, elles atteignent de fortes dimensions et devaient être, vraisemblablement, lacées entre elles " .

L'armure du XIV" siècle est donc composite (Fig. 2) et, selon l'endroit, la protection est assurée par des pièces métalliques rigides ou par un tissu de mailles entrelacées (Fig. 3). C'est ce que montrent les sceaux, les miniatures et d'autres monuments figurés, ainsi que l'examen de nombreuses restitutions ${ }^{12}$ ou des armures d'époque, telles celles déposées

13 Stephen V. Grancsay: Les armures, adapt. de R. T. Charles, Paris, Edit. des Deux Coys d'Or, 1965, pp. 42-44.

1 Bengt Thordeman: Armour from the ballle of Visby, 1361, Stockholm, Kungl. Vitterhets Hist. och Antik. Akademie, 1939, 2 vols.

12 Le Musée de l'Armée, à Paris, montrait de bonnes reconstitutions d'armes et d'armures dans les salles Charlemagne, Bayard, etc. Ces reconstitutions ainsi que ces salles ont disparu lors des modifications qu'a souffert de Musée. 


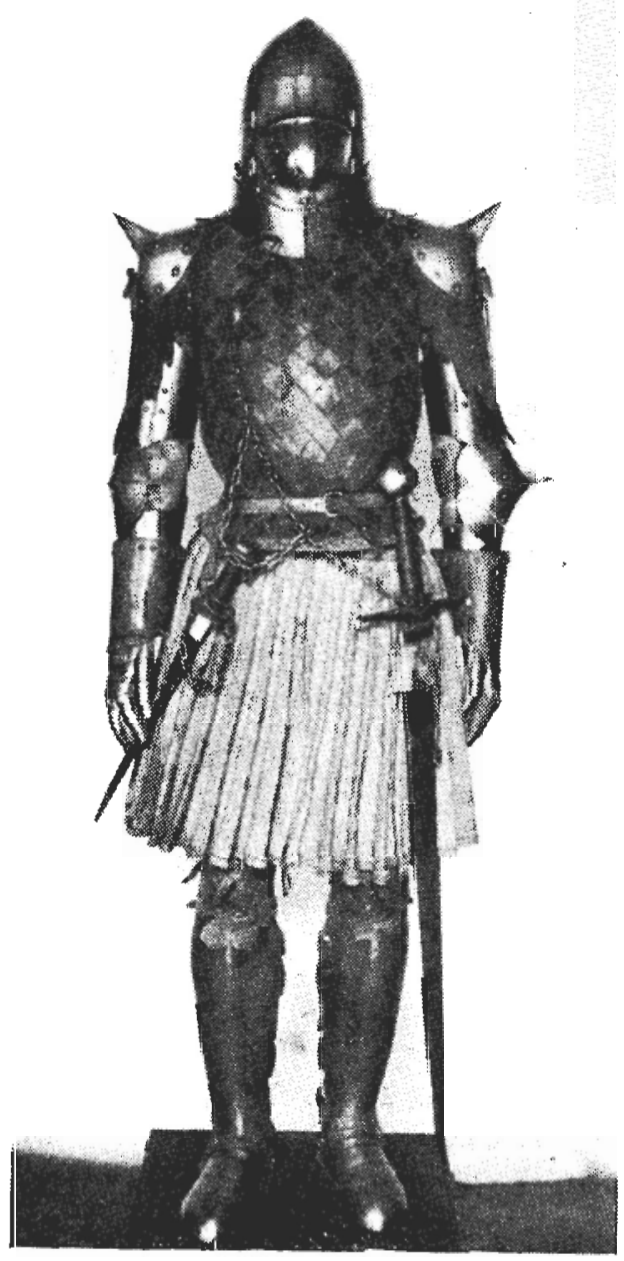

FIG. 3.-Armure composite du $X I V^{*}$ siècle. Reconstitution qui existait il n'y a guère d'années au Musée de l'Armée à Paris. Le personnage êtait censé appartenir au règne du roi Jean II, dit le Bon ou le Brave (13501364). Les plates renforçent la protection aux endroits sensibles: épaules, coudes, bas des jambes, etc. L'épée et la dague sont retenues par les chaînes d'armes. Sur la tête, un grand bassinet. (Phot. Archives photographiques.)

au Musée de Chartres, à la Tour de Londres (armure dite de Rodolphe IV duc d'Autriche), etc. Par-dessus l'armure, on porte une longue tunique, parfois rembourrée, qui aide à amortir le choc des projectiles ennemis, et masque les solutions de continuité entre les plates (Fig. 4).

L'écu, maintenant moins nécessaire, voit diminuer ses dimensions. D'abord il prend la forme d'un triangle équilatéral, dont les bords laté- 
raux peuvent être curvilignes. Puis il perd sa pointe aigüe et tend vers le carré ou le rectangle. C'est la targe, bouclier de forme complexe, très étudiée qui, à la fin du siècle voit croître sa largeur en même temps que sa hauteur diminue et qui peut s'agrémenter d'une cannelure servant à loger le bras et l'avant-bras, ce qui aide à détourner les coups.

L'usage du heaume se maintient presque jusqu'à la fin du siècle. On peut voir ainsi le heaume de guerre jadis porté par Sir Richard Pembridge, mort en 1375, déposé aujourd'hui au Royal Scottish Museum d'Edimbourg (cote 489), ou celui du Prince Noir († 1376) conservé à la cathédrale de Canterbury où il fut enterré. Ces heaumes pèsent environ $3 \mathrm{~kg}$.

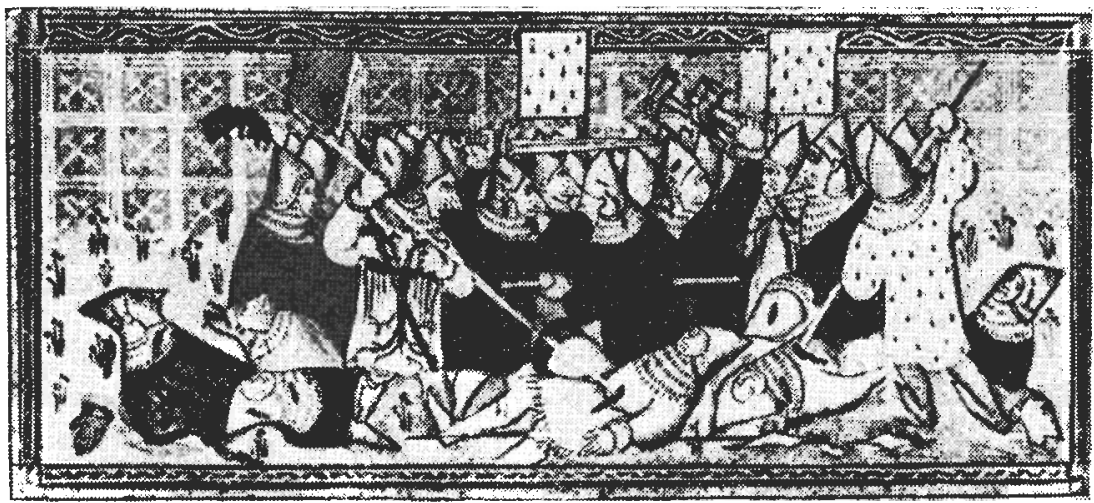

Fig. 4.-Balaille d'Auray, 1364. «D'après la Chronique de Du Guesclin», manuscrit conservé à la Bibliothèque Nationale de Paris. Au premier plan et à gauche, Du Guesclin, reconnaissable à ses armoiries - un aigle bicéphale-_ peintes sur sa poitrine. Les combattants, revêtus de la lourde cotte et portant le bassinet à visière aigüe, sont armés de lances, d'épées et de marteaux. (P. Martin: Armes el armures, p. 88 , fig. 73.)

chacun, un tel poids explique leurs abandon pour la guerre et on les réserve pour les tournois ou la parade. Ils peuvent être alors surmontés d'un cimier, superstructure de carton, de cuir ou de bois léger, représentant un animal héraldique, une ramure, etc. Pour le combat, on porte un casque qui coiffe le crâne et s'unit au reste de l'armure par un camail, large manchon de mailles qui couvre le bas de la nuque, le col et, parfois, les épaules ainsi que le haut de la poitrine; une visière mobile permet de découvrir le visage sans enlever le casque (Fig. 4). C'est le bassinet ou bacinet, tel celui qu'on peut voir aujourd'hui à la Tour de Londres (cote IV-470); provenant du château de Churburg, dans le Sud du Tyrol, il a 
sû être fait dans un atelier milanais vers 1380-1390. D'autres sont conservés au Musée de l'Armée (cotes H 15 à H 21) ainsi que dans diverses armureries d'Europe. Lorsque la partie haute du camail est remplacée par des plaques d'acier cerclant le col ainsi que la gorge, on a le grand bassinet, caractéristique des règnes de Jean le Bon et de Charles V, dont un bel exemplaire est déposé au Musée de l'Armée (cote H 24).

La lance a une hampe d'environ $3 \mathrm{~m}$. armée d'un fer en forme d'alène ou de dague à section triangulaire ou losangée, long d'une trentaine de centimètres, et qui lui est très fortement fixé afin de résister aux chocs contre les plate de l'adversaire; une rondelle d'acier, plate ou convexe, protège la main. Dès l'époque de Philippe le Bel, l'épée n'est plus utilisée pour la taille et, désormais, elle apparait réservée aux coups d'estoc. Sa lame, étroite, aigüe, très rigide, renforcée par une arête médiane, est longue d'environ $1 \mathrm{~m}$. Dans les combats à pied, on l'empoigne à deux mains, comme une lance de fer et, même maniée de taille, cette lourde barre peut fausser une plate ou rompre un membre. Près du pommeau, un orifice ou un anneau permet de passer la chaine d'armes qui rattache l'épée à l'armure. De la sorte, si l'épée lui est arrachée des mains, le combattant peut la rattraper et des chaînes analogues servent à retenir la dague, le heaume, etc. (Fig. 2 et 3 ).

Certains semblent avoir désespéré de pouvoir entamer les armures avec la lance ou l'épée d'estoc. C'est pourquoi ils ajoutent à leur armement une masse ou un marteau d'arçon à manche court, entièrement métallique et pourvu d'un bec à l'une de ses extrémités, tandis que d'autres choissisent une bacbe d'arçon garnie, elle aussi, d'un bec au talon. Avec ces armes il est possible d'assener des coups de volée extrêmement violents, capables de percer les plates.

Pour les rencontres à pied, on utilise généralement, soit une lance dont la hampe, raccourcie, ne mesure que cinq pieds environ (à peu près $1,50 \mathrm{~m}$.), soit une darde, arme d'hast au manche relativement court muni d'un fer aigü à double tranchant, soit enfin, d'une bache d'armes, bache de Créqui ou bache à dague, longue d'environ $1,50 \mathrm{~m}$., maniée à deux mains et qui oppose à son tranchant une pointe ou un croc tandis qu'une longue pointe la prolonge vers le haut.

Du côté anglais, outre les chevaliers armés de toutes pièces, il y a vers 1340 les bobelars, qui tirent leurs nom de celui des petits chevaux ou bobyns qu'ils montent. Porteurs d'une épée, d'un couteau et d'une lance, ainsi que d'un gambison et d'un simple bassinet, ces hobelars constituent une véritable cavalerie légère, sans équivalent parmis les forces françaises de l'époque, quoiqu'on puisse la trouver au XIII" siècle ou même avant, et qu'elle fasse sa réapparition au $X^{*}$ comme auxiliaire des gendarmes. D'ailleur les hobelars s'éclipseront bientôt des armées 
anglaises et, une quinzaine d'années après, il n'y en a pas ni dans la chevauchée entreprise à travers le Languedoc par le Prince Noir (1355) ni lors de la bataille de Poitiers (1356).

Infanterie.--Les gens de pied portent une brigandine mais, plus souvent une courte cotte de mailles entrelacées ou un gambison, avec ou sans manches. Parfois on les remplace par un simple vêtement de cuir sur lequel on applique des bandes de cuir treillisée. Quelques plates (cubitières, etc.) peuvent renforcer le tout. La tête est couverte par un casque conique ou sphérique, avec ou sans nasal et losque le casque est muni de larges ailes, tombantes comme celles d'une capeline, on a le chapeau de Montauban qui protège du tir plongeant des flèches ennemies.

Comme armes offensives, on trouve la hache, la masse ou le marteau dont le long manche permet d'atteindre un cavalier. On porte aussi l'épée et, parfois, on se sert d'un modèle long et lourd, que l'on manie à deux mains et qui sera souvent utilisé par les Suisses au XVI siècle. La dague, qui semble avoir fait son apparition à la fin du XIII“ siècle, se répand au XIV' parmis les cavaliers et les piétons. Le perce-mailles est un roide poignard en forme d'alène, d'un pied de long, permettant d'achever l'homme d'armes jetté à terre. Quant au couteau à plates, sa lame très fine, peut s'introduire entre les pièces de l'armure.

Les lanciers ou sergents à lance et bouclier, sont munis d'une lance raccourcie ou d'un cousil _-courte hampe pourvue d'un fer semblable à un sabre-baionnette — ou, enfin, des armes d'hast du type déjà connu aux siècles précédents: guisarme, faucbard, vouge, etc. Sans essayer d'éclaircir des dénominations que les auteurs contemporains semblent avoir employé indistinctement, il suffira de dire qu'elles consistent en une hampe, longue d'environ $2 \mathrm{~m}$, armée d'un fer large et long, propre à frapper de taille ou à trancher les jarrets des chevaux de l'adversaire, munie d'une pointe permettant de s'en servir d'estoc et d'un ou plusieurs crochets pour désarçonner les ennemis ou pour arrêter une arme glissant le long de la hampe.

Les gens de trait sont, principalement, les arcbers, du côté anglais, et les arbalétriers, du côté français.

Les Anglais, eux, employent le grand arc ou lang bow, qui est une arme d'origine galloise, fort redoutable, mesurant environ $2 \mathrm{~m}$. et donc les meilleurs sont faits en bois d'if. Si le tir est dirigé vers le haut, en suivant une trajectoire parabolique, la flèche -longue de $1 \mathrm{~m}$.- peut atteindre 30 ou $40 \mathrm{~m}$. de hauteur, passer par-dessus n'importe quel obstacle et, en touchant le sol, transpercer encore une planche de bois de $0,03 \mathrm{~m}$. d'épaisseur. Tiré en plein fouet, l'arc porte à plus de $150 \mathrm{~m}$. et, à cette distance, sa flèche perce une planche de 0,025 à $0,03 \mathrm{~m}$. La balle 
du Winchester 44, le fusil qui conquit l'Ouest nord-américain, traverse, à 4,50 m. de la bouche de l'arme, 13 planches de sapin de $0,021 \mathrm{~m}$. d'épaissur chacune, placée à $0,021 \mathrm{~m}$. l'une de l'autre ${ }^{13}$. Comme un archer entrainé peut décocher une douzaine de flèches à la minute, tandis qu'un arbalétrier ne peut envoyer que deux carreaux, on a une arme très puissante et à tir rapide. A la bataille de Crécy (1346), les chevaliers français et les arbalétriers qui les soutenaient de leur tir, succombent sous la grêle des flèches ennemies.

Lors de cette bataille, les cavaliers anglais étaient descendu de leurs montures pour encourager et couvrir les hommes de pied. Comme, de plus, leurs archers ont l'habitude de ficher devant eux de longs pieux qui embarassent les chevaux ennemis et les laissent à la merci des flèches, une nouvelle tactique surgit aux environ de 1350: celle de la cavalerie démontée. Au moment du combat, les cavaliers mettent pied à terre et, marchant lourdement, aidés au besoin par leurs valets, ils s'avancent vers l'adversaire, les flèches ayant peu d'effet contre les plates. Cette tactique est illogique en soi puisqu'elle prive la cavalerie de l'appoint du cheval qui constitue sa raison d'être, néanmoins elle sera adoptée presque partout et se prolonger'a jusqu'au milieu du $\mathrm{XV}^{\mathrm{e}}$ siècle. Accessoirement, on assiste à l'abandon de la house ou protection du cheval puisque celui-ci, écarté au moment de la lutte, est laissé en arrière et ne sert que pour la poursuite... ou pour la fuite.

Les arbalétriers forment la majorité des gens de trait de l'armée française. Ce sont, pour la plupart, des Génois engagés comme mercenaires, qui se servent de l'arbalète à croc, lançant des traits —dondaines, viretons, carreaux ou garrots - plus trapus que la flèche de l'arc ${ }^{14}$. Pour bander cette arbalète, on se penche vers le sol en tenant l'arme verticale, le pied est engagé et maintenu dans l'étrier qui termine le fût, la corde passée par un croc ou crochet pendu à la ceinture, puis on se relève brusquement; la corde ainsi tendue pénètre dans une encoche y reste fixée pendant que le tireur vise et n'en est expulsée que par l'action de la détente. Pour pouvoir préparer leur arme à l'abri, les arbalétriers sont munis de pavois, boucliers rectangulaires, hauts de $1 \mathrm{~m}$., larges de la moitié, faits de bois légèr doublé de peau et pourvus d'une forte cannelure verticale pour y passer un pieu permettant de les fixer au sol. D'autres fois, le pavois est attaché sur le dos du tireur à qui il suffit de se retourner pour être à couvert. Comme, malgré sa légèreté, le poids et la

13 Manufacture Françalse d'Armes et Cycles: Tarif-Album, Saint-Etienne, 1910 , p. 124.

${ }^{14}$ SiR R. PAYNe-GnLlwey: The crossbow medieval and modern... treatise on the balista and catapult of the Ancients... the Turkish bow, London, The Holland Press, 1958, xxii, 328, 24, 23 p. 
manoeuvre du pavois risquent d'être embarassants, un pavesier est chargé de ce soin et, en principe, on compte un pavesier pour deux arbalétriers.

Quant aux armes à feu portatives, elles ne s'emplorons vraiment qu'à partir du XIV ciècle finissant et ne sont citées ici que pour mémoire.

\section{L'ARMEMENT AU XV $\mathrm{XV}^{\mathrm{e}}$ SIÈCLE ${ }^{15}$}

Cavalerie.-Au $X V^{c}$ siècle, le torse est couvert de plates métalliques -plastron, pansière, dossière - sur lesquelles s'articulent, à leur tour, les spalières ou épaulières, les brassards, les cubitières et les gantelets, pour les membres supérieurs, ainsi que les cuissards, les genonillères, les jambières et les solerets pour les inférieurs; la tête étant défendue par l'armet, de forme sphérique ou ovoïde, qui se substitue au heaume et au bassinet. L'armure composite du XIV ${ }^{c}$ siècle a donc disparu, reemplacée par un ensemble de plaques rigides s'articulant les unes sur les autres comme la carapace de certains crustacés. C'est l'armure de plain ou armure de plates complètes, dont les musées offrent de nombreux exemples et qui a été popularisée par l'illustration et par... le cinéma. Son poids oscile entre 25 et $30 \mathrm{~kg}$., auquel il faut ajouter 45 à $50 \mathrm{~kg}$. pour le poids de la protection du cheval - celle-ci réapparaissant dans la seconde moitié du $X V^{c}$ siècle avec le nouvel emploi de la bête pour le combat - et quelques 75 à $80 \mathrm{~kg}$. pour le poids du cavalier. On arrive ainsi à un total de 150 à $160 \mathrm{~kg}$. et le cheval de bataille devient maintenant un véritable animal de labeur, lourd, épais, puissant. À titre de comparaison, rappelons qu'à la veille de la guerre de 1914-1918, l'équipement du cuirassier français (armes, vivres, selle) ne dépasse guère le poids de $30 \mathrm{~kg}$.

L'armure est généralement faite sur mesure afin que les articulations puissent jouer parfaitement, mais les armuriers ont aussi des armures semi-terminées ou prêtes à porter dont les prix sont, naturellement fort inférieurs. Les plaques d'acier sont rendues très dures par un long battage à froid et les plattners (batteurs de plates) d'Augsbourg, d'Innsbruck ou de Nuremberg ainsi que leurs confrères italiens ou anglais, français ou espagnols, réalisent des travaux remarquables ${ }^{16}$. Au début du $X V^{e}$

${ }^{15}$ Aux ouvrages indiqués à la note 7 , on ajoutera: marquis R. DE Belleval: Du costume militaire des Français en 1446, Paris, Aubry, 1866, viii, 91 p.

${ }^{16}$ Des répertoires de marques d'arnuriers les plus fameux, ont été donnés à plusieur reprises. Voir, entre autres, celui établi par G. R. Maurice Maindron: Les armes, Paris, Quantin, 1890 (Bibliothèque de l'Enseignement des Beaux Arts), pp. 338-343. 
siècle, la surface de la plupart des armures est simplement polie (barnois blanc), puis on commence à les orner de gravures et de damasquinures; cette décoration est, souvent, d'une haute valeur artistique mais elle ne se répand vraiment qu'au $X V I^{\circ}$ siècle, au moment où les armes à feu aménent une réduction du nombre de pièces portées par l'homme de guerre et où l'armure complète ne subsiste que chez les grands seigneurs, à la fois vêtement de combat et de parade. Plus tard, les officiers supérieurs sont les seuls qui portent l'armure entière, se conformant ainsi aux ordres royaux et, au milieu du XVII ${ }^{*}$ siècle encore, Louis XIII continuera à l'exiger.

Rappellons que les vêtements défensifs de choix peuvent être à l'épreuve, à botte cassée, de baute botte, de toute botte, etc., lorsqu'ils ont résisté au tir des viretons décochés par l'arbalète à treuil, ou à demiépreuve lorsque ce tir n'est effectué qu'avec un arc ou une arbalète ordinaire. Dans le premier cas, deux poinçons de contrôle leurs sont apposés par le maître qui les a forgé, tandis que dans le second, ils ne reçoivent qu'un seul poinçon.

Le cavalier lourd peut avoir une masse ou un marteau d'arçon mais ses armes propres sont l'épée d'estoc et la lance. La première continue à être une lame longue d'environ $1 \mathrm{~m}$., roide et très aigüe. La lance, elle, s'est transformée pour devenir, maintenant, un véritable bélier armé d'un fer à pans coupés. Poutre de $5 \mathrm{~m}$. de long., au profil renflé, son diamètre peut atteindre $0,10 \mathrm{~m}$. et il se retrécit à l'endroit où se pose la main. La rondelle, de grandes dimensions, est conique comme une trompette. Pour rendre le choc de la lance plus puissant, on place un collier de billettes d'acier autour de la hampe, entre la rondelle et la prise de main. Au moment de charger, le cavalier s'affirme sur les étriers, s'allonge sur l'encolure du cheval, avance légèrement le corps du côté gauche, couche la lance et serre le collier de billettes contre l'arret de cuirasse. Cet arret, parfois appelé érronéement faucre, est une sorte de crochet entouré de plomb ou de bois tendre, fixé sur la plastron de la cuirasse ${ }^{17}$. Lorsque la lance bute contre un obstacle, les billetes s'incrustent dans le revêtement de l'arret, les trois éléments -lance, homme, cheval - ne font plus qu'un et l'obstacle est balayé.

Le choc d'une telle cavalerie est irrésistible mais ses possibilités de manoeuvre sont restreintes et elle ne peut guère agir que dans des conditions favorables. Force est donc de lui adjoindre des auxiliaires moins lourdement harnachés qui forment une sorte de cavalerie légère. Cette cavalerie légère, dont la disparition dans les armées françaises et anglai-

17 François Buttun: La lance et l'arrêt de cuirasse, in: «Archaeologia», LondonOxford, t. 99, 1965. pp. 77-205. 
ses n'avait été que transitoire, va alors s'affermir et son emploi ne cesseia de s'accroitre dans les siècles à venir. Ces auxiliaires ont le corps protégé par une brigandine garnie de cubitières et par des genouillères. Sur la tête ils portent une salade, casque rond d'origine italienne, muni d'ur couvre-nuque allongé ainsi que de jouées verticales. Parfois, le casque est complété par une visière fixe percée d'une fente pour la vue de sorte qu'en marche, il suffit de le rejeter quelque peu sur la nuque pour découvrir le visage; au moment du combat, on le rabat vers l'avant et la visière masque la face. Comme armes offensives, l'épée d'estoc ainsi que l'arc ou le coustil; fort souvent, une hache au fer triangulaire, pouvant être utilisée comme arme de volée ou de taille.

Infanterie- - «Au début de la guerre de Cent ans, l'immense majorité des combattants se répartit en deux catégories: les gens d'armes et les gcns de pied. On ne peut manquer d'être frappé par l'importance de ces derniers qui constituent fréquemment au moins la moitié des efectifs. En 1482, la répartition est la suivante: cavalerie, $38 \%$; infanterie, $44 \%$; troupes de garnison, $13 \%$; artillerie et génie, $5 \%$. Là aussi l'infanterie joue un róle majeur. Cependant, l'examen des deux périodes extrêmes masque l'évolution qui s'est produite dans l'intervalle. En effet, de 1350 à 1450 environ, l'infanterie subit une éclipse marquée et ce fut seulement au cours de la deuxième moitié du $15^{\circ}$ siècle qu'elle retrouva progressivement la place qu'elle tenait durant la première moitié du $14^{\circ}$ siècle. Par ailleur, deux types militaires nouveaux son apparus: d'une part, les utilisateurs d'armes à feu - canonniers, coulevriniers- dont le nombre est passé de quelques dizaines à la fin du $14^{\text {t }}$ siècle à plusieurs centaines vers 1480; d'autre part, les gens de trait à cheval —arbalétriers et surtout archers- deux fois moins nombreux que les hommes d'armes vers 1400 , deux fois plus nombreux à partir des années $1430 \gg{ }^{18}$.

Les piquiers ont une défense de corps analogue à celle des cavaliers légers: brigandine ou gambison, quelques plates, un bouclier rectangulaire et une salade à couvre-nuque vertical, généralement prolongée par un camail. Lorsque le casque est diminué, de façon à ne couvrir que le dessus du crâne, des sortes de disques métalliques posés sur les côtés, prorègent les oreilles. Comme armes offensives, ces piquiers portent l'épée, le couteau à plates et les armes d'hast déjà vues. Ils font parfois usage du fléau, de la plommée ou du goupillon, ensembles de boules métalliques ou de saumons hérissés de pointes ou de têtes de diamant, fixés à des chaïnes reliées à un manche. En les faisant tournoyer, on porte des coups très violents, capables de fausser les plates, d'assommer les hom-

". Ph. Contaminf: Guerre, Elat el sociélé... (cf. note 1), p. 533. 
mes ou de rompre les membres. Ces coups sont fort difficiles à parer parceque les chaïnes contournent le bouclier ou l'arme mise en travers. Par contre, seuls les hommes très exercés peuvent s'en servir d'une façon efficace, sans se blesser les uns les autres.

Les gens de trait, eux, ont habituellement un jacque, vêtement fait d'une trentaine d'épaisseurs de toile piquée sur un fond de cuir et renforcé, au besoin, de plates. Un casque similaire à celui des autres hommes de pied, une épée et, souvent, une arme d'hast complétent la panoplie qui, naturellement, comprend aussi le grand arc ou une arbalète. Parmis ces dernières signalons celles dites à treuil, à moufles ou à girelle, qui apparaissent alors représentées dans les monuments figurés et dont l'arc d'acier, court et roide, est bandé au moyen de moufles et d'un petit treuil amovible. Dans l'arbalète à cric ou à cry, la corde est tendue par l'action d'un cric, mécanisme qui, on l'a déjà dit, fait son apparition au $\mathrm{XV}^{\mathrm{e}}$ siècle (Fig. 5).

La force de pénétration de ces armes est devenue très considérable et la courte flèche ainsi envoyée peut «transpercer ung demy pied de porte» ${ }^{19}$, c'est-à-dire un madrier d'environ $0,15 \mathrm{~m}$. d'épaisseur. Rien d'étonnant alors si, à partir de 1484 «les capitaines furent autorisés à inclure dans les 200 archers que comptait une compagnie de 100 lances, 15 ou 20 arbalétriers exercés» ${ }^{20}$.

Quant aux armes à feu «les historiens ont donné, pour les premières armes à feu portatives que les textes aient mentionnées, les dates de 1364 à Perouse, de 1381 à Augsbourg» ${ }^{21}$. La coulevrine dont une belle figuration est fournie par le Bellifortis de Konrad Kyeser ${ }^{22}$, ne représente qu'un antécédent et on pourrait aussi bien la ranger parmis les pièces d'artillerie légère. Par contre, les bastons à feu ou traits à feu, constituent vraiment des armes individuelles. Ils sont formés d'un tube en fer

${ }^{1}$ Octavien de Saint Gelais et Andry de. ia Vigne: Le Vergier d'bonneur... de l'entreprise et voyage de Napples... s. 1. n. d. (Bibliothèque Nationale, Réserve 4. $\mathrm{L} \mathrm{b}^{2 *}, 15 \mathrm{~A}$ ), fol. e VI.

20. Ph. Contamine: Guerre, Étal el société... (cf. note 1), p. 461.

21 Bertrand Gille: Les XVr et XVI siècles..., in: «Histoire générale des techniques» (cf. note 4), vol. II, p. 96.

${ }_{22}^{22}$ La miniature qui orne le fol. 104 v. du manuscrit de Göttingen a été souvent reproduite, notamment par: Marcellin Berthelot: Histoire des macbines de guerre... (cf. note 6), p. 388, fig. 90; BerTRAND G1lle: Les ingénieurs de la Renaissan ce (cf. note 3), p. 53; Bertrand Gillf. Les XV* et XVI siècles..., in: "Histoire générale des techniques» (cf. note 4), vol. II, p. 90, fig. 42; J.-F. Finó: L'artillerie e'n France à la fin du Moyen Âge, in: Gladius, t. XII, 1975, p. 13 et pp. 23-24, fig. 5. Une autre figuration, appartenant au manuscrit dit de Besançon, est reproduite par Victor GAY: Glossaire arcbéologique du Moyen Äge et de la Renaissance, Paris, Libr. de la Société Bibliographique, 1887-1928, article «Canon», vol. I, p. 273. 
forgé, parfois à sept ou huit pans, long d'une trentaine de centimè̀res et prolongé par une tige d'environ $1 \mathrm{~m}$., garnie d'un bouton: la tige passe sous l'aiselle du tireur et, si elle est plus courte, s'appuye sur la cuidasse. Un croc, dans le bas du canon, permet aux cavaliers de prendre appui sur une autre tige, fixée à la selle ou aux fantassins d'en faire autant sur une fourche plantée devant eux. Lorsque le tube de fer est par trop mince,

FIG. 5.-Arbalètes du $X V^{c}$ siècle. A gauche, une arbalète à moufles. À droite, une autre à cric. Les mécanismes de tension sont nettement visibles et rendent toute explication superflue. (A bist. of technol., vol. II, p. 722.)

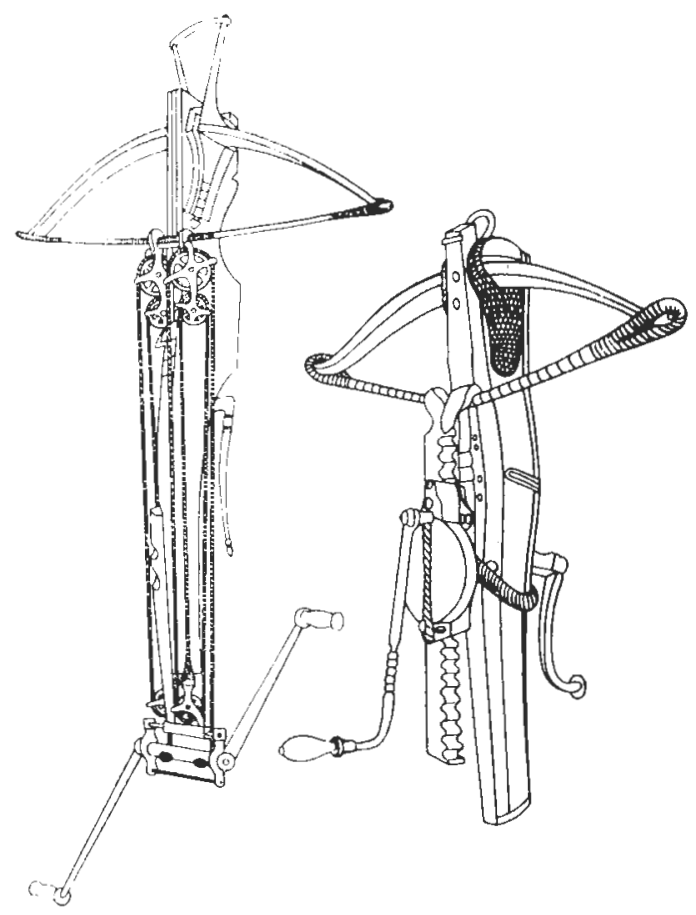

il peut être doublé d'un autre tube, de bois celui-ci. Un baston d'époque est visible au Musée de l'Arsenal Royal de Copenhague (Våbensalen, gulvmontre 1). Brisé par éclatement, il a été trouvé à Vedelspang (Danemark), sur l'emplacement d'un château bâti en 1416 et détruit en 1426, ce qui permet de dater l'objet avec assez de précision (Fig. 6).

Bientôt le tube devient plus long et la tige est remplacée par une sorte de crosse, large et lourde, munie d'une entaille qui, pour le tir, s'emboite sur l'épaule du soldat: coulevriniers du règne de Charles VII. 
L'arme, chargée de poudre grossière et d'un projectile —carreau d'arbalète, balle de plomb, etc.- est tenue de la main gauche qui sert à pointer. L'ayant ainsi stabilisée, le tireur approche une mèche allumée de la lumière - petit orifice foré dans la partie arrière du tube- et le feu se transmet à la poudre, manoeuvre qui apparait clairement représentée dans de nombreuses miniatures contemporaines ${ }^{23}$.

Le tir est lent et imprécis. Les éclatements et les ratés sont fréquent. Néanmoins, les armes à feu s'imposent, le nombre d'hommes qui en portent ne cesse de s'accroitre et la bataille de Pavie (1525) est gagnée par les espagnols, en grande partie grâce leurs arquebusiers. L'arbalète

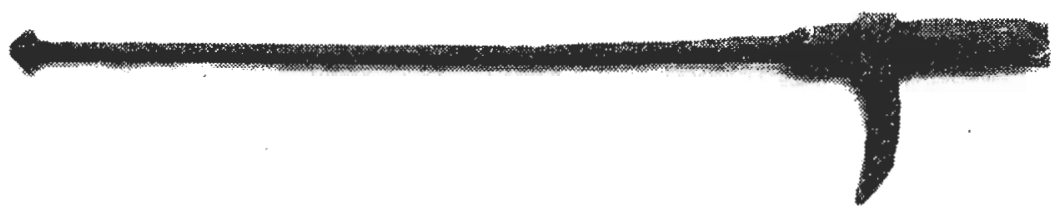

FIG. 6.-Báton à féu du premièr quart du XVe siècle. L'objet est aujourd'hui déposé au Musée de ['Arsenal Royal cle Copenhague (gulvmontre 1). On voit le tube de fer servant a contenil la charge de poudre et le projectile, la tige qui prolonge ce tube et qui se termine par un bouton, enfin le croc métallique au moyen duquel on appuyait l'arme pour le tir. (Phot. Musée.)

va donc s'effacer peu à peu devant elles et, finalement, une ordonnance rendue en 1567 par le roi Charles IX, en supprimera l'usage dans les armées françaises.

Arillerie et génie.-L'artillerie dite classique (trébuchets et mangonneaux) ne se distingue pour ainsi dire pas de celle utilisée aux siècles précédents ${ }^{24}$. Quant à l'artillerie à feu, dans cette même revue nous en avons dit quelques mots à propos de sa naissance, de son évolution en France à la fin du Moyen Âge, etc. ${ }^{25}$; inutile donc d'y revenir ici. Il suffit de souligner que, sauf un nombre réduit de techniciens chargés de la fabrication et de la conduite des pièces, la défense et le service de celles-

${ }^{23}$ VICTOR GAY: Glossaire archéologique (cf. note 22), article «arquebuse», vol. I, p. 73; article «hacquebute», vol. II, p. 8, etc.

24 J.-F. Finó: Machines de jet médiévales, in: Gladues, t. X, 1972, pp. 25-43.

25 J.F. Finó: L'artillerie en France... (cf. note 22). 
ci sont assurés par des soldats pris à l'unité la plus proche, tandis que leurs transport est le fait de charretiers et pionniers spécialement levés pour une campagne déterminée. Les hommes retenus en permanence sont donc peu nombreux et ceci ne fera que s'aggraver au cours des siècles. Au XVIII", le Royal Artillerie, seul régiment de l'arme existant à l'époque, ne compte guère que 5.000 hommes, tandis que l'effectif des armées dépasse, à plusieurs reprises, 300.000; le pourcentage est donc inférieur à celui indiqué pour les armées françaises en 1482. Une situation analogue règne aussi dans ce que l'on pourrait appeler le génie.

\section{EFFECTIFS ET COMMANDEMENT}

Les effectifs, quoique supérieurs à ceux des siècles précédents, apparaisseni fort restreints à nos yeux ${ }^{26}$. Pour la France, en 1340, Philippe VI de Valois aurait eu, au total $\$ 20.000$ hommes d'armes et 16.700 gens de pied dont un bon nombre d'arbalétriers... Durant l'été 1451, Charles VII entretint nettement plus de 20.000 combattants» ${ }^{27}$. Remarquons toutefois que, bien qu'il lcs solda, Philippe VI ne pouvait compter sur de pareilles forces que pendant quel jues semaines tandis que Charles VII dispose d'une armée permanente. Pour l'Angleterre, le nombre d'hommes engagés pour le combat semble être de l'ordre de 18.000 pour Crécy (1346), de 10.000 pour Poitier (1356) et, en 1415, de 11.000 pour Azincourt ${ }^{28}$.

L'état de guerre étant quasi continuel, l'aide financière des villes et des provinces, qui permet de payer l'armée, le devient aussi. De plus, on assiste à la formation des Grandes Compagnies, des Écorcheurs, etc., fléaux à propos desquels il manque une étude d'ensemble ${ }^{29}$ malgré qu'il y a près de vingt ans que R. Fawtier en signalait la nécéssité ${ }^{30}$. Ces deux facteurs, ainsi que d'autres que l'on pourrait citer, aboutissent à la permanence de l'armée sous Charles VII. Pour la cavalerie, une ordonnance de 1445, institue 15 Compagnies d'Ordonnance ou Ordonnances du Roi.

2" Les ouvrages essenticls son: PH. Contramine: Guterre, État et société... (cf. note 1), pussim; Histoire des institutions fronçaises au Moyen Äge, publice sous la direction de Ferdinand Lot et de Robert Fawtier, vol. II (Paris, Presses Universitaires de France, 1958), pp. 511-535. «L'al'mée»; Ferdinand Lot: L'art militaire et les armées an Moyen Âge et dans le Proche Orient, Paris, Payot, 1946, 2 vols.

${ }_{27}$ Pir. Contamine: Gucrre, État et société... (cf. note 1), pp. 70 et 314.

2* Ferdinand Lo't: L'art militaire... (cf. note 26), vol. I, pp. 344-348 et 359-364; vol. II, pp. 13-14 et 484 .

2) A. TueTey: Les Écoicheurs sous Chanles VII, Montbéliard, H. Barbier, 1874, 2 vols.; ne traite qu'une partie de la question.

${ }^{30}$ Histoire des insitutitions... (cf. note 26), vol. II, p. 522, note 1. 


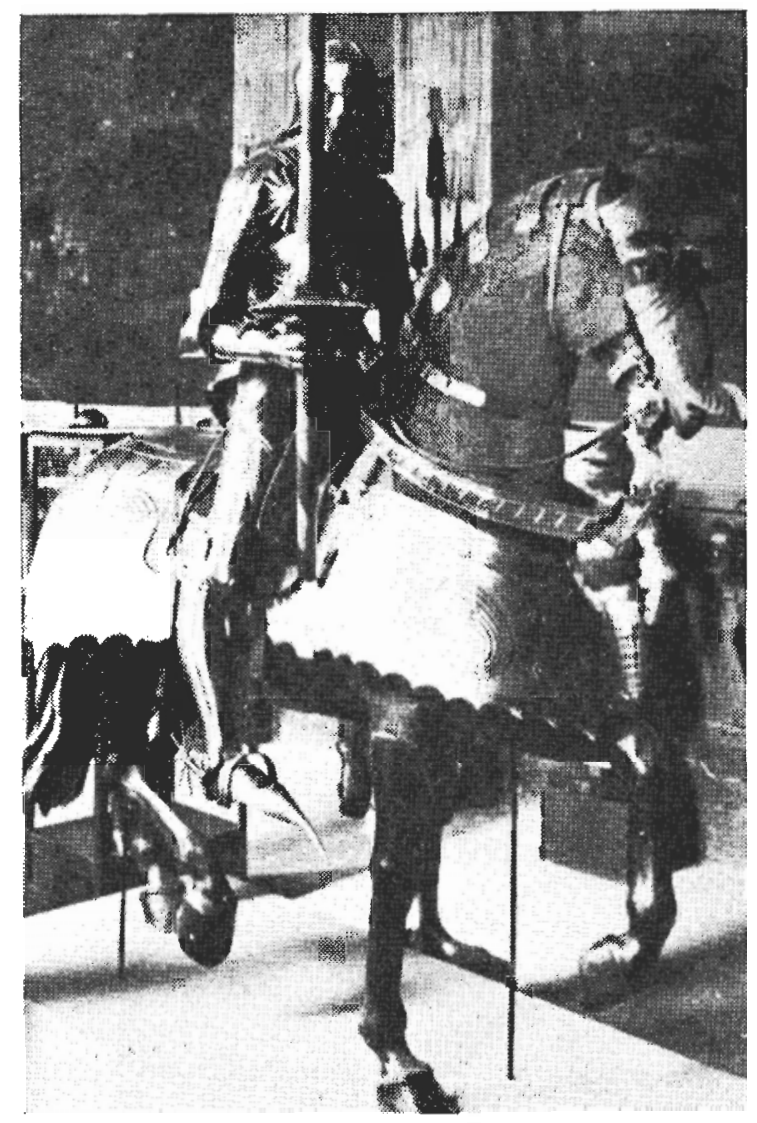

FIG. 7.-Gendarme des Compagnies d'Ordonnance. Armure authentique existante au Musée de l'Armée. Elle êtait exhibée dans la Salle Pierrefonds, ainsi appellée parceque les armes et armures qu'on y voyait avaient jadis appartenus à la collestion privée de Napoléon III, au château de Pierrefond; confisqué lors de la déposition de l'Empereur, l'ensemble fut transporté à Paris, et le nom de la salle (aujourd'hui disparu) en rappelait l'origine. Le gendarme est entièrement couvert de fer et sa tête est protegée par une salade cont la visière masque la face. Le cheval est, lui-aussi cuirassé et un tissu de mailles entrelacées en couvre l'encolure. La lance, reconsituée, n'êtait plus exposée dans les dernières années. (Phot. Archives photographiques.) 
La Compagnie se compose — du moins en théorie- de 100 lances fournies ou garnies; chaque lance est formée d'un gendarme, portant l'armure complète et commandans le groupe (Fig. 7), de deux archers et d'un coustillier ou coustillieur, plus légérement armés, ainsi que d'un page et d'un valet, auxiliaires non combattants chargés des chevaux et d'aider le gendarme à s'approcher de l'ennemi lorsqu'on applique la tactique de la cavalerie démontée. Tous ces hommes se déplacent à cheval mais, au moment du combat, les archers et le coustillier mettent pied à terre pour mieux manier leurs armes. Cette cavalerie est forte d'environ 6 à 7.000 combattants puisqu'aux 1.500 lances d'Ordonnance il faut ajouter les 500 lances du Languedoc et que, parfois, le valet est remplacé par un troisième archer. Régulièrement soldée, casernée dans les châteaux du Roi ou logée chez l'habitant aux frais du Trésor royal, elle n'a pas de rivale en Europe et elle constituera, jusqu'au XVIc siècle, le noyau de l'armée française. Pour l'infanterie, on essaye bien, par une ordonnance de 1448, de l'organiser aussi d'une façon régulière. Un ou plusieurs hommes, exercés au tir de l'arc ou de l'arbalète, doivent être fournis par chaque paroisse. Exempts de taille, d'où leur nom de Francs-Archers, ces quelques 8.000 hommes forment une sorte de milice. En temps de paix, ils vivent de leurs métiers civil. En cas de guerre, on les convoque et ils sont gratifiés d'une solde. L'institution ne rendant pas les services que l'on attendait d'elle, force fut de recourrir aux mercenaires (Suisses, Allemands, etc.), pratique qui se perpétuera jusqu'aux temps modernes ${ }^{31}$.

Quant au commandent en chef, il revient de plein droit au souverain mais, dans certaines occasions, il est impossible à celui-ci de l'exercer effectivement, il est remplacé alors par le connétable ${ }^{32}$. On trouve «à l'échelon inmédiatement inférieur, les lieutenants et capitaines généraux désignés par le roi [et qui] exercent leur commandement et leurs pouvoirs dans une région déterminée» ${ }^{33}$. Parallèlement à ces chefs régionaux, il existe les commandants d'une ou plusieurs armées destinées à entreprendre des campagnes plus ou moins longues et lointaines. Audessous, viennent les chefs de montre qui sont, à la fois, le chef militaire de leurs hommes et la personne avec laquelle s'entendent les trésoriers de guerre. Puis les chevetaines, capitaines, dizainiers, etc., qui vont s'imposer peu à peu, favorisés par le Pouvoir Royal et dont l'essort mar fuera la transformation de l'armée qui cesse d'être une institution féodale pour devenir un organisme d'état.

${ }^{31}$ Hisloire des institutions... (cf. note 26), vol. II, pp. 523-535.

32 Il s'agit ici du véritable connétable ou connétable de France, non point de ceux qui, comme Pierre Pillonay en 1358, sont à la tête d'un groupe de gens de guerre.

${ }_{33}^{3}$ Ph. Contamine: Guerre, État et société... (cf. note 1), p. 75. 